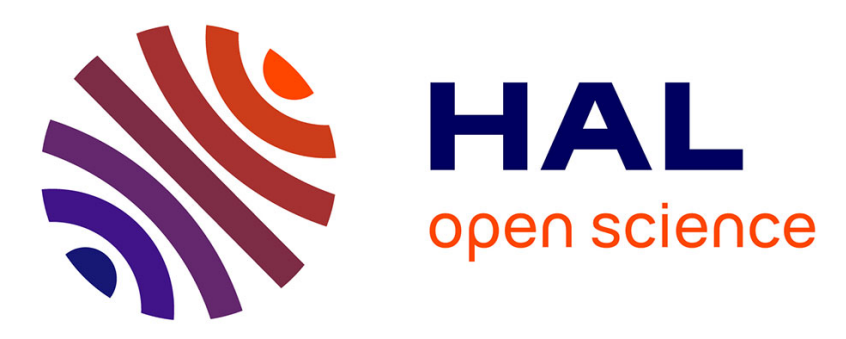

\title{
Using Closed n-set Patterns for Spatio-Temporal Classification
}

\author{
Saulius Samulevicius, Yoann Pitarch, Torben Bach Pedersen
}

\section{To cite this version:}

Saulius Samulevicius, Yoann Pitarch, Torben Bach Pedersen. Using Closed n-set Patterns for SpatioTemporal Classification. 16th International Conference on Data Warehousing and Knowledge Discovery (DaWaK 2014), Sep 2014, Munich, Germany. pp. 274-287. hal-01399862

\section{HAL Id: hal-01399862 \\ https://hal.science/hal-01399862}

Submitted on 21 Nov 2016

HAL is a multi-disciplinary open access archive for the deposit and dissemination of scientific research documents, whether they are published or not. The documents may come from teaching and research institutions in France or abroad, or from public or private research centers.
L'archive ouverte pluridisciplinaire $\mathbf{H A L}$, est destinée au dépôt et à la diffusion de documents scientifiques de niveau recherche, publiés ou non, émanant des établissements d'enseignement et de recherche français ou étrangers, des laboratoires publics ou privés. 


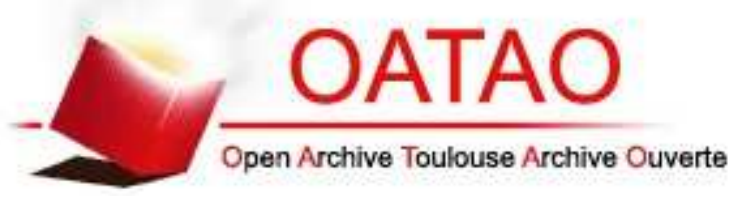

\section{Open Archive TOULOUSE Archive Ouverte (OATAO)}

OATAO is an open access repository that collects the work of Toulouse researchers and makes it freely available over the web where possible.

This is an author-deposited version published in : http://oatao.univ-toulouse.fr/ Eprints ID : 15178

The contribution was presented at $\mathrm{DaWaK} 2014$ :

http://www.dexa.org/dawak2014

To cite this version : Samulevicius, Saulius and Pitarch, Yoann and Pedersen, Torben Bach Using Closed n-set Patterns for Spatio-Temporal Classification. (2014) In: 16th International Conference on Data Warehousing and Knowledge Discovery (DaWaK 2014), 1 September 2014 - 5 September 2014 (Munich, Germany).

Any correspondence concerning this service should be sent to the repository administrator: staff-oatao@listes-diff.inp-toulouse.fr 


\title{
Using Closed n-set Patterns for Spatio-Temporal Classification
}

\author{
S. Samulevičius ${ }^{1}$, Y. Pitarch ${ }^{2}$, and T.B. Pedersen ${ }^{1}$ \\ 1 Department of Computer Science, Aalborg University, Denmark \\ \{sauliuss, tbp\}@cs.aau.dk \\ 2 Université of Toulouse, CNRS, IRIT UMR5505, F-31071, France \\ pitarch@irit.fr
}

\begin{abstract}
Today, huge volumes of sensor data are collected from many different sources. One of the most crucial data mining tasks considering this data is the ability to predict and classify data to anticipate trends or failures and take adequate steps. While the initial data might be of limited interest itself, the use of additional information, e.g., latent attributes, spatio-temporal details, etc., can add significant values and interestingness. In this paper we present a classification approach, called Closed n-set Spatio-Temporal Classification (CnSC), which is based on the use of latent attributes, pattern mining, and classification model construction. As the amount of generated patterns is huge, we employ a scalable NoSQL-based graph database for efficient storage and retrieval. By considering hierarchies in the latent attributes, we define pattern and context similarity scores. The classification model for a specific context is constructed by aggregating the most similar patterns. Presented approach $\mathrm{CnSC}$ is evaluated with a real dataset and shows competitive results compared with other prediction strategies.
\end{abstract}

Keywords: Pattern mining, time series, classification, prediction, context, latent attributes, hierarchy.

\section{Introduction}

Huge volumes of sensor data are collected from many different sources. As a result, enormous amounts of time series data are collected constantly. Raw data without post-processing and interpretations has little value, therefore multiple algorithms and strategies have been developed to deal with knowledge and behavior discovery in the data, i.e., classification, pattern mining, etc. The discovered knowledge allows predicting the behavior of the system being monitored, i.e., identify potential risks or trends, and perform optimization according to them. When the data distribution is stationary and the values are discrete, this is thus a classification problem. Latent attributes, i.e., attribute values inferred from the raw data, has proven to be a good strategy for enhancing data mining performance. Latent attributes can represent different types of information such as temporal, e.g., day and hour, spatial, e.g., POI, spatio-temporal, e.g., events, 
etc. Such attributes form a context for the raw data. Similar raw data records will (typically) have similar contexts.

In this paper we present a spatio-temporal classification approach, called Closed n-set Spatio-Temporal Classification (CnSC) which is based on closed n-set pattern mining in hierarchical contextual data and subsequent aggregation of the selected mined patterns. The proposed approach extends traditional classification approaches in several directions. Firstly, we utilize hierarchies in the latent attributes, i.e., if none of the mined patterns match a specific context, we instead use the most similar (according to the hierarchical level). Secondly, pattern mining operations are the most time-consuming part and require special treatment. We thus propose persistent pattern storage using a NoSQL graph database (DB) and an effective scheme for mapping the mined patters to graphs. These solutions support efficient pattern storage and retrieval. $\mathrm{CnSC}$ is experimentally evaluated using the real-world sensor dataset from a mobile broadband network. The evaluations show that properly configured CnSC, outperforms the existing solutions in terms of classification accuracy.

The remainder of the paper is organized as follows. In Section 2 we present the relevant related work. Background and problem definitions are stated in Section 3. In Section 4 we describe CnSC. The graph database solutions and mappings are described in Section 5. We experimentally evaluate CnSC in Section 6 and conclude with our contributions and future work in Section 7.

\section{Related Work}

Different approaches considering patterns have been analyzed in a number of papers the recent years. The two main application areas where patters have been used are prediction and classification. Spatio-temporal pattern-based prediction often analyzes trajectory patterns of the moving user. User profiles defined using historical locations enable estimate future, e.g., places that potentially will be visited in the city [10], opponent actions in games [5], or mobility patterns for personal communication systems [15]. In this paper we analyze the prediction potential using mined patterns.

A survey of recent pattern-based classification papers is presented in [1], where classification strategies are compared considering a) efficient pattern storage and operation methods, e.g., use of trees or graphs; b) pattern selection and model construction for classification, e.g., iterative pattern mining for the optimal model construction. Most of the pattern-based papers address a common problem, i.e., huge amount of mined patterns, which requires efficient pattern operations. Rule-based classifiers C4.5 [11] and CMAR [8] store patterns in decision trees for higher efficiency. In $\mathrm{CnSC}$ we consider graph database [6], which allows efficiently manipulate mined patterns using SQL like queries. Top-K most similar pattern use for the pattern-based classification is a common strategy $[1,14]$. Other strategies, such as, similarity between patterns [2] or emerging patterns [4], are used for the optimal pattern selection. In this paper we define similarity metric for pattern and context which incorporates hierarchical attribute structure. 
The selected patterns further has to be aggregated into a single classification model [8]. To achieve higher classification results, pattern selection, feature extraction, and model construction can be done iteratively [1], i.e., pattern mining is continued until the classification model meets required threshold. In this paper we run non-iterative process and optimize classification results by considering hierarchical structures in the data. Most often classification of the unknown values returns a default class [9] value. In CnSC this problem is solved considering hierarchical structures.

\section{Background and Problem Definition}

\subsection{Data Format}

Let $\mathcal{S}$ be a set of time series such that each $S_{i}$ in $\mathcal{S}$ is on the form $S_{i}=\left(i d_{i}, s e q_{i}\right)$ where $i d_{i}$ is the time series identifier and $s e q_{i}=\left\langle s_{i, 1}, \ldots, s_{i, N}\right\rangle$ is the sequence of records produced by $i d_{i}{ }^{1}$. Each $s_{i, j}=\left(t i d_{i, j}, v_{i, j}\right)$ conveys information about the timestamp, $t i d_{i, j}$, and the value, $v_{i, j}$. The time series identifiers are denoted by $i d$ and the set of timestamps are denoted by tid. Seasonality can often be observed in the time series. However, since we aim at exploiting intra-seasonal patterns to model time series behaviors, this seasonality aspect is disregarded by splitting time series according to an application-dependent temporal interval $T$, e.g., one day, one week. This results in a bigger set of shorter time series, denoted by $\mathcal{S}^{T}$, such that each element is on the form $S_{i}^{k}=\left(i d_{i}^{k}, \operatorname{seq}_{i}^{k}\right.$, sid $\left._{i}^{k}\right)$ where $s i d_{i}^{k}$ is the section identifier of $S_{i}^{k}$ and indicates which part of the original time series, $S_{i}$, is represented by it, i.e., $s i d_{i}^{k}=k$.

Considering latent attributes in the mining process has often proven to be a good strategy to enhance the result quality [12]. Latent attributes can be temporal, e.g., the sensor value captured during the night, spatial, e.g., is the sensor near to some restaurants, or spatio-temporal, e.g., is there any traffic jam next to a sensor. The set of latent attributes, denoted by $\mathcal{A}=\left\{A_{1}, \ldots, A_{M}\right\}$, can be derived from the time series identifier and the timestamp of a record using a function denoted $M a p$, i.e., Map $: i d, t i d \rightarrow \mathcal{A}$. Latent attributes can be hierarchical ${ }^{2}$. The hierarchy associated with the attribute $A_{i}$ is denoted by $H\left(A_{i}\right)=A_{i, 0}, \ldots, A_{i, A L L_{i}}$ where $A_{i, 0}$ is the finest level and $A_{i, A L L_{i}}$ is the coarsest level and represents all the values of $A_{i}$. $\operatorname{Dom}\left(A_{i}\right)$ is the definition domain of $A_{i}$ and $\operatorname{Dom}\left(A_{i, j}\right)$ is the definition domain of $A_{i}$ at level $A_{i, j}$. An instance $a \in \operatorname{Dom}\left(A_{1}\right) \times \ldots \times \operatorname{Dom}\left(A_{M}\right)$ is called a context and it is a low level context if $a \in \operatorname{Dom}\left(A_{1,0}\right) \times \ldots \times \operatorname{Dom}\left(A_{M, 0}\right)$. Some notations are now introduced: $U p\left(a_{i}, A_{i, j}\right)$ returns the unique generalization (if it exists) of $a_{i}$ at level $A_{i, j}$; $\operatorname{Down}\left(a_{i}, A_{i, j}\right)$ provides the set of specializations (if it exists) of $a_{i}$ at level $A_{i, j}{ }^{3}$; $N b L e a v e s\left(a_{i}\right)$ returns the number of specializations of attribute value $a_{i}$ at the

${ }^{1}$ For ease of reading and whiteout loss of generality, we assume that each time series has the same length $N$.

${ }^{2}$ No restriction is made on the type of hierarchies.

3 These two functions can be straightforwardly extended to contexts. 
finest level $A_{i, 0}$; and, given an attribute value $a_{i}, L v\left(a_{i}\right)$ returns its hierarchical level. Finally, given a context, denoted by $a$, the function LowLevelContext $(a)$ returns the set of low level contexts which are a specialization of $a$.

A user-defined discretization function Disc is introduced to map time series values into a set of $L$ classes, denoted by $C=\left\{c_{1}, \ldots, c_{L}\right\}$. Finally, to provide the information about how often a context, denoted by $a$, is associated to a class value, $c$, we introduce the function Support that is defined as $\operatorname{Support}(a, c)=$ $\left\{\operatorname{sid}_{i}^{j} \mid \exists\left(t i d_{i, k}^{j}, v_{i, k}^{j}\right)\right.$ s.t. $\operatorname{Map}\left(T_{i}^{j}, t_{i} d_{i, k}^{j}\right)=a$ and $\left.\operatorname{Disc}\left(v_{i, k}^{j}\right)=c\right\}$. The input dataset structure is now formally defined.

Definition 1 (Input dataset structure). Let $\mathcal{S}$ be a time series dataset, $T$ be a temporal interval, $\mathcal{A}$ be a set of latent attributes, and $C$ be a set of classes derived from the time series value discretization. The input dataset, denoted by $\mathcal{D}=\left\{d_{1}, \ldots\right\}$, is such that each $d_{i}$ is on the form $d_{i}=\left(a_{i}, c_{i}\right.$, Supp $\left.p_{i}\right)$ where $a_{i}$ is a context, $c_{i} \in C$ is a class value, and $\operatorname{Supp}_{i}=\operatorname{Support}\left(a_{i}, c_{i}\right)$.

Example 1. Assuming $T=1$, time series $S_{1}$ and $S_{2}$ are split in 2 shorter time series, i.e., $S_{1}$ into $S_{1}^{1}$ and $S_{1}^{2}$, and $S_{2}$ into $S_{2}^{1}$ and $S_{2}^{2}$, see Fig.1(a). The set of latent attributes $\mathcal{A}=\{A, B\}$ with associated hierarchies is shown in Fig.1(b) and Fig.1(c). Generalization of attribute $a_{11}$ at level $A_{1}$ is $U p\left(a_{11}, A_{1}\right)=a_{1}$ and specialization of attribute $a_{1}$ at level $A_{0}$ is Down $\left(a_{1}, A_{0}\right)=\left\{a_{11}, a_{12}\right\}$.

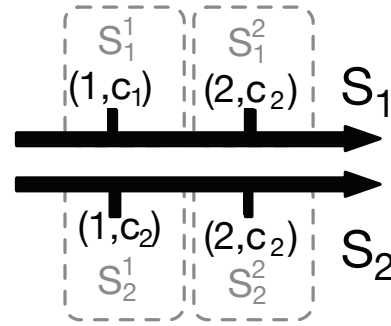

(a)

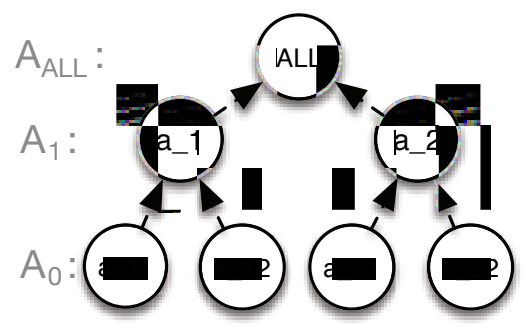

(b)

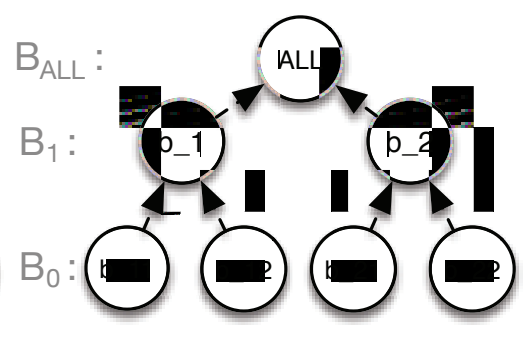

(c)

Fig. 1. (a) Time series splitting strategy with $T=1$; (b) Hierarchy associated with the latent attribute $A$; (c) Hierarchy associated with the latent attribute $B$

\section{2 n-ary Closed Sets}

Our classification approach relies on n-ary closed sets which are described here. Let $\mathcal{A}^{c}=A_{1}^{c}, \ldots, A_{n}^{c}$ be a set of $n$ discrete-valued attributes whose domains are respectively $\operatorname{Dom}\left(A_{1}^{c}\right), \ldots, \operatorname{Dom}\left(A_{n}^{c}\right)$ and $R$ be a n-ary relation on these attributes, i.e., $R \subseteq \operatorname{Dom}\left(A_{1}^{c}\right) \times \ldots \times \operatorname{Dom}\left(A_{n}^{c}\right)$. One can straightforwardly represent this relation as a (hyper-)cube where the measure $m$ associated with the cell $\left(a_{1}, \ldots, a_{n}\right)$, such that $a_{i} \in A_{i}^{c}$ with $1 \leq i \leq n$, equals 1 when $R\left(a_{1}, \ldots, a_{n}\right)$ holds and 0 otherwise. Intuitively, a closed n-set can thus be seen as a maximal subcube of 1's. More formally, a n-set $H=\left\langle X_{1}, \ldots, X_{n}\right\rangle$ such that $X_{i} \subseteq \operatorname{Dom}\left(A_{i}^{c}\right)$ 
is a closed n-set iff (a) all elements of each set $X_{i}$ are in relation with all the other elements of the other sets in $R$, and (b) the $X_{i}$ sets cannot be enlarged without violating (a). In [3], the algorithm DATA-PEELER has been proposed to efficiently solve the pattern mining problem. In this paper, this approach will be used to mine n-ary closed sets.

We consider $\mathcal{A}^{c}$ as the union of latent attributes and the class attribute. It thus enables the discovery of patterns that can be seen as a maximal spatio-temporal context having the same class value. Since DATA-PEELER does not consider hierarchical attributes, only low-level latent attribute values are considered during the mining phase. Moreover, even if n-ary closed patterns do not convey any information about the support of a pattern, it is possible to incorporate it very straightforwardly by considering the Support function as an attribute of $\mathcal{A}$.

Definition 2 (Pattern). A pattern is in the form $P=\left(A_{1}^{P}, \ldots A_{n}^{P}, C^{P}, S u p p^{P}\right)$ such that $A_{i}^{P} \subseteq \operatorname{Dom}\left(A_{i, 0}\right), C^{P} \subseteq C$ and $\operatorname{Supp}^{P}=\bigcap \operatorname{Support}(a, c)$ with $a \in\left(A_{1}^{P} \times \ldots \times A_{n}^{P}\right)$ and $c \in C^{P}$. The set of patterns is denoted by $\mathcal{P}$.

Finally, DATA-PEELER allows to set some thresholds to prune the search space. Among them, the minSetSize parameter is a vector whose length is the number of attributes, and specifies the minimum size of each attribute set that compose a pattern. In this study, this parameter is used to fulfill two goals. First, mined patterns must include at least one class value, i.e., $\left|C^{P}\right|>0$ for all $P \in \mathcal{P}$. Second, as shown in our experiment study analysis, it can significantly reduce the number of extracted patterns.

Example 2. Let us assume the following Map functions: $\operatorname{Map}\left(S_{1}, 1\right)=\left(\left\{a_{11}\right.\right.$, $\left.\left.a_{21}\right\},\left\{b_{12}, b_{21}\right\}\right), \operatorname{Map}\left(S_{1}, 2\right)=\left(\left\{a_{21}\right\},\left\{b_{22}\right\}\right), \operatorname{Map}\left(S_{2}, 1\right)=\left(\left\{a_{12}\right\},\left\{b_{11}, b_{12}\right\}\right)$, and $\operatorname{Map}\left(S_{2}, 2\right)=\left(\left\{a_{11}, a_{12}\right\},\left\{b_{11}\right\}\right)$. Input for DATA-P EELER is shown in Fig. 2 (left). For instance, the first four cells in the left table are associated with $\operatorname{Map}\left(S_{1}, 1\right)$. Its class is $c_{1}$ and after splitting $T=1,\left(1, c_{1}\right)$ belongs to $S_{1}^{1}$. Running DATA-PEELER with parameter minSetSize $=\left(\begin{array}{llll}1 & 1 & 1 & 1\end{array}\right)$ mines patterns with minimum one value of each attribute, see in Fig. 2 (right). For instance, $P_{1}$ means that the context $\left(a_{12}, b_{11}\right)$ is associated with the class value $c_{2}$ in the two time intervals, i.e., 1 and 2.

\begin{tabular}{c|cc}
$S_{1}$ & $S_{2}$ & $P_{1}=\left(\left\{a_{12}\right\},\left\{b_{11}\right\},\left\{c_{2}\right\},\{1,2\}\right)$ \\
$\left\{a_{11}\right\},\left\{b_{12}\right\},\left\{c_{1}\right\},\{1\}$ & $\left\{a_{12}\right\},\left\{b_{11}\right\},\left\{c_{2}\right\},\{1\}$ & $P_{2}=\left(\left\{a_{11}, a_{12}\right\},\left\{b_{11}\right\},\left\{c_{2}\right\},\{2\}\right)$ \\
$\left\{a_{11}\right\},\left\{b_{21}\right\},\left\{c_{1}\right\},\{1\}$ & $\left\{a_{12}\right\},\left\{b_{12}\right\},\left\{c_{2}\right\},\{1\}$ & $P_{2}=\left(\left\{a_{21}\right\},\left\{b_{22}\right\},\left\{c_{2}\right\},\{2\}\right)$ \\
$\left\{a_{21}\right\},\left\{b_{12}\right\},\left\{c_{1}\right\},\{1\}$ & $\left\{a_{11}\right\},\left\{b_{11}\right\},\left\{c_{2}\right\},\{2\}$ & $P_{3}=\left(P_{4}=\left(\left\{a_{11}, a_{21}\right\},\left\{b_{12}, b_{21}\right\},\left\{c_{1}\right\},\{1\}\right)\right.$ \\
$\left\{a_{21}\right\},\left\{b_{21}\right\},\left\{c_{1}\right\},\{1\}$ & $\left\{a_{12}\right\},\left\{b_{11}\right\},\left\{c_{2}\right\},\{2\}$ & $P_{4}=\left(c_{5}=\left(\left\{a_{12},\left\{b_{11}, b_{12}\right\},\left\{c_{2}\right\},\{1\}\right)\right.\right.$ \\
$\left\{a_{21}\right\},\left\{b_{22}\right\},\left\{c_{2}\right\},\{2\}$ & & $P_{5}=(1)$
\end{tabular}

Fig. 2. (left) The dataset $\mathcal{D}$ which serves as input for DATA-PEELER ; (right) n-ary closed set extracted from $\mathcal{D}$. 


\subsection{Problem Definition}

Given a set of closed n-sets, $\mathcal{P}$, extracted from $\mathcal{D}$ with minSetSize $=\boldsymbol{v}$ and an evaluation dataset, $\mathcal{D}_{E}$, with unknown class values, our objective is two-fold:

1. Accuracy. Given a context, denoted by $a \in \mathcal{D}_{E}$, performing an accurate classification requires (1) identifying an appropriate subset of $\mathcal{P}$ and (2) combining these patterns to associate the correct class to the context $a$.

2. Efficiency. Pattern mining algorithms often generate a huge amount of patterns. Dealing with up to millions of patterns require efficient storage and fast pattern retrieval techniques.

\section{Pattern-Based Classification}

We first provide a global overview of $\mathrm{CnSC}$ and then detail its two main steps:

(1) finding patterns that are similar to the context we would like to predict and

(2) combining these patterns to perform the prediction.

\subsection{Overview}

For each context, denoted by $a$, the first step is to identify similar patterns. The similarity between a pattern $P$ and a context $a$ is formally defined in Subsection 4.2. In a few words, similarity between $P$ and $a$ will be total if there exists a perfect matching between the context $a$ and the contextual elements of $P$. Otherwise, the similarity will be high if $P$ involves elements that are hierarchically close to the members of context $a$, i.e., few generalizations are needed to find the nearest common ancestors of each $a_{i}$ in $a$. Searching for these similar patterns might be particularly tricky when dealing with a huge amount of patterns. We propose an algorithm to efficiently retrieve these patterns. The similar patterns are aggregated to support the final decision according the following principle. Patterns are grouped depending on their class value(s). For each class, a score is calculated based on both the support and similarity of associated patterns. The class with the highest score is then elected.

\subsection{Getting the Most Similar Patterns}

Our similarity measure relies on the hierarchical nature of latent attributes and makes use of the nearest common ancestor definition.

Definition 3 (Nearest common ancestor). Let $a_{i}$ and $a_{i}^{\prime}$ be two elements of $\operatorname{Dom}\left(A_{i, 0}\right)$. The nearest common ancestor of $a_{i}$ and $a_{i}^{\prime}$, denoted by nca $\left(a_{i}, a_{i}^{\prime}\right)$, is minimum attribute at level $A_{i, j}$ which generalizes $a_{i}$ and $a_{i}^{\prime}$ :

$$
n c a\left(a_{i}, a_{i}^{\prime}\right)=U p\left(a_{i}, A_{i, j}\right)=U p\left(a_{i}^{\prime}, A_{i, j}\right) \text { with } j=\underset{x \in\left[0, A L L_{i}\right]}{\arg \min }(x)
$$


Definition 4 (Similarity measure). Let $P=\left(A_{1}^{P}, \ldots A_{n}^{P}, C^{P}\right.$, Supp $\left.^{P}\right)$ be a pattern and $a^{\prime}=\left(a_{1}^{\prime}, \ldots, a_{n}^{\prime}\right)$ be a low-level context. The similarity between $P$ and $a^{\prime}$, denoted by $\operatorname{Sim}\left(a^{\prime}, P\right)$, is defined as:

$$
\operatorname{Sim}\left(a^{\prime}, P\right)=\frac{1}{\prod_{i=0}^{n} \min _{a_{i} \in A_{i}^{P}}\left(\left|\operatorname{Down}\left(n c a\left(a_{i}^{\prime}, a_{i}\right), A_{i, 0}\right)\right|\right)}
$$

Example 3. Consider the hierarchies in Fig.1 and pattern $P_{4}$ in Fig. 2, we estimate the similarity between the context $a^{\prime}=\left(a_{22}, b_{12}\right)$ and $P_{4} . n c a\left(a_{22}, a_{11}\right)=$ $A_{A L L}$, i.e, $\left|\operatorname{Down}\left(A_{A L L}, A_{0}\right)\right|=4$, nca $\left(a_{22}, a_{21}\right)=a_{2}$, i.e, $\left|\operatorname{Down}\left(a_{2}, A_{0}\right)\right|=2$, and $n c a\left(b_{12}, b_{12}\right)=b_{12}$, i.e, $\left|\operatorname{Down}\left(b_{12}, B_{0}\right)\right|=1$, thus $\operatorname{Sim}\left(a^{\prime}, P_{4}\right)=\frac{1}{2 * 1}=0.5$.

Searching for these most similar patterns can be problematic when dealing with huge amount of patterns. Interestingly, by analyzing our similarity function behavior, an efficient algorithm can be designed to retrieve the patterns. The most similar patterns are obviously patterns having the same context as the one we are interested in. To avoid cases where no pattern perfectly matches the context, i.e., no classification could be performed, patterns being similar enough are also considered. To this aim, a user-defined numerical threshold, denoted by minSim, is introduced to retain all the patterns having similarity greater or equal than minSim. There are as many choices as the number of latent attributes. Since we aim at finding the most similar patterns, it is necessary to minimize the denominator of the similarity function. Thus, the generalization must be performed on the attribute value whose generalization has the smallest number of leafs. This one-by-one generalization process is repeated iteratively until no more similar pattern is found. The set of the extracted most similar patterns is denoted by $\mathcal{P}$ (a, minSim). Algorithm 1 formalizes this process.

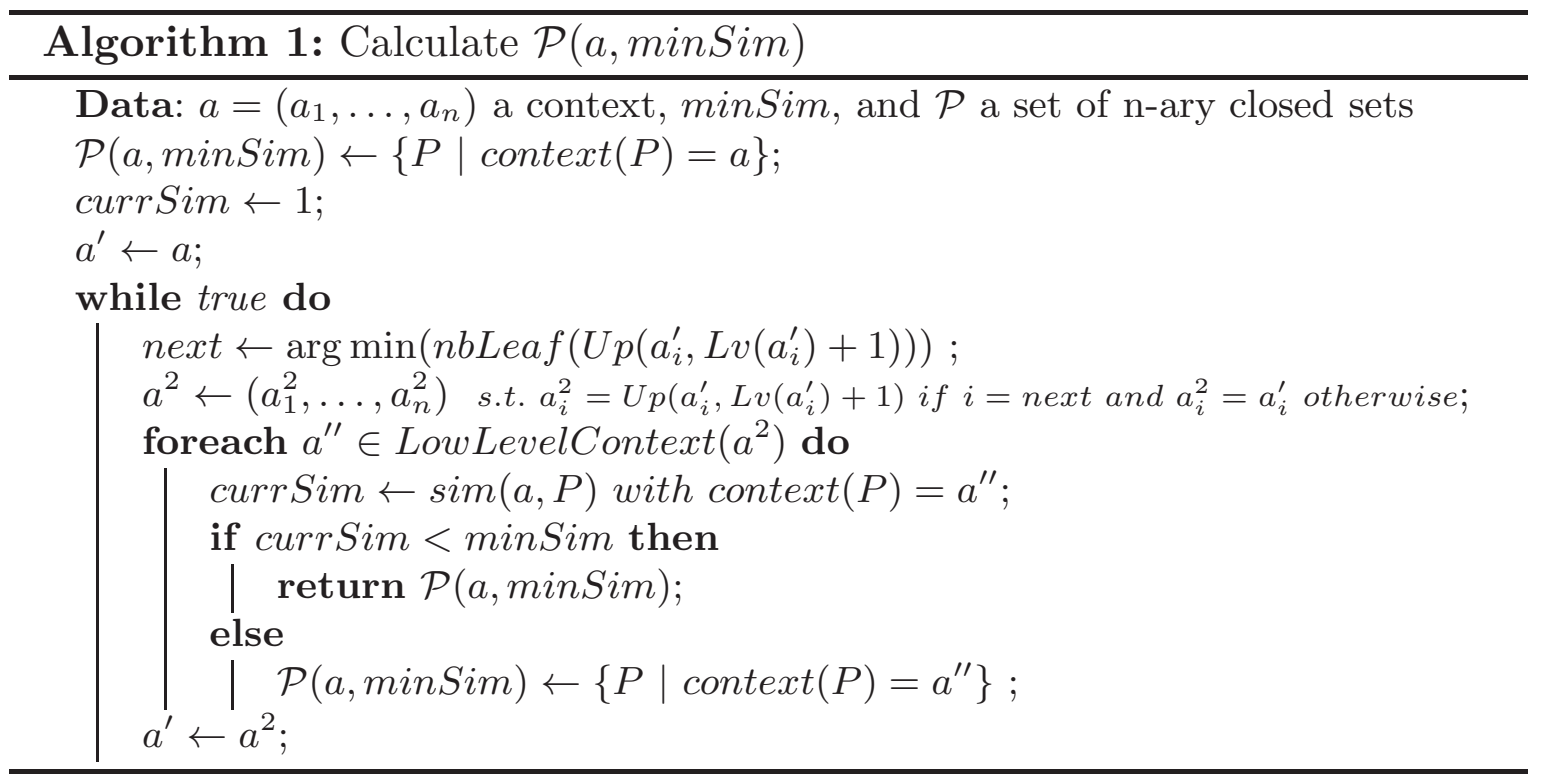




\subsection{Combining Patterns}

Once similar patterns have been found, the classification can be performed. For each class, $c \in C$, a score is calculated based on the patterns associated with $c$. This score is based on both the support of the pattern and its similarity with the context to classify. Algorithm 2 formalizes this process.

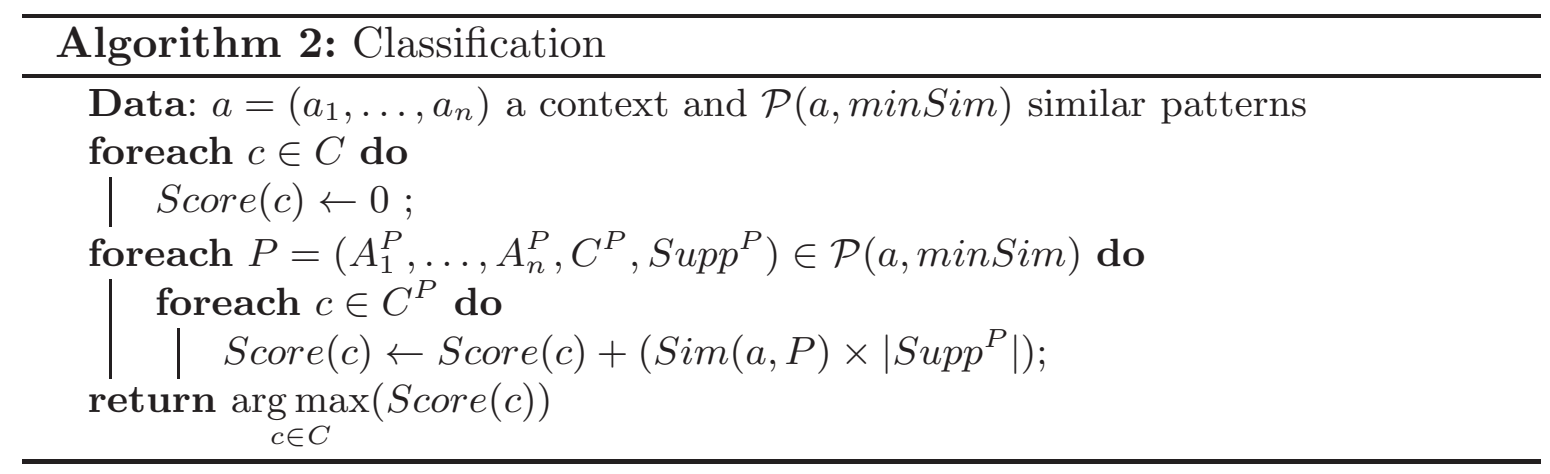

Example 4. Classification process of the context $a=\left(a_{22}, b_{12}\right)$ is now illustrated considering the set of patterns shown in Fig. 2 and minSim $=0.2$. Only $P_{4}$ is associated with the class value $c_{1}$. The score associated with this class value is thus $0.5 \times 1$, i.e., the product between the similarity between the context and the pattern and the size of the pattern support set size. For class value $c_{2}$, its score is $\frac{1}{4} \times 1=0.25$ (coming from $P_{5}$ ) since the others patterns have a similarity with a being lower than minSim, e.g, Sim $\left(a, P_{1}\right)=\frac{1}{8}<\operatorname{minSim}$. The class value $c_{1}$ will be the one predicted here.

\section{$5 \quad$ Efficient and Compact Pattern Storage}

\subsection{Requirements}

A well-known result in pattern mining, as well as in DATA-PEELER, is the generation of a huge amount of patterns, i.e., millions or billions of n-ary closed sets. Such output is definitely non-human-readable and, even worse, it can significantly reduce its potential for being used in more complex analysis. In this study, two requirements should be met to make CnSC tractable:

- Persistent storage. As stated in the experiment results, DATA-PEELER is the most time consuming step within the whole process. For this reason, it is run only once. To avoid pattern recompilation in case of breakdown, it is preferable not to store patterns in main memory but rather persistently.

- Fast point-wise pattern query. As detailed in the previous section, the most critical operation of our method is searching for the most similar patterns of a given context. To do so, Algorithm 1 extensively queries the pattern set using point-wise queries in order to test if a pattern exists. Therefore, the data structure should be optimized for such queries. 


\subsection{Graph Definition}

Our method is based on a graph structure for two main reasons. First, hierarchical and membership relationships can be naturally expressed in a graph-based fashion. Second, the rise of NoSQL databases, and especially graph databases, enables a persistent storage of graphs. Such graph databases are particularly of interest in our scenario since we can benefit from indexing techniques, and powerful and efficient SQL-like data manipulation languages. We now detail our model choices.

Vertices are of one single type and represent values of the latent attributes. The set of vertices is denoted $V$. Given $v \in V$, the function label $(v)$ returns the attribute value represented by $v$. Two types of relationship co-exist in the graph structure. First, an arc from $v_{1}$ to $v_{2}$ exists if $v_{2}$ is a direct generalization of $v_{1}$. Second, there exists an edge between two data nodes if (1) they do not belong to the same attribute and (2) if there exists at least one pattern where these two nodes co-occur. Some properties are attached to these edges: the class, a list of pattern identifier(s) and a list of pattern support(s). Note that this modeling enables the existence of $L$ edges between two vertices where $L$ is the number of classes. The pattern modeling is less intuitive and will be discussed in Section 5.4. We now formally define these relationship categories.

Definition 5 (The $\boldsymbol{I S} \boldsymbol{A}$ relationship). Let $v$ and $v^{\prime}$ be two vertices. There exists a directed so-called IS A relationship between $v$ and $v^{\prime}$, denoted by $e=$ $\left(v, v^{\prime}\right)$, if $\operatorname{Up}(\operatorname{label}(v), \operatorname{lv}(\operatorname{label}(v))+1)=\operatorname{label}\left(v^{\prime}\right)$. The set of IS A relationships is denoted by $E_{I}$.

Definition 6 (The CO-OCCURING relationship). Let $v$ (resp. $v^{\prime}$ ) be a vertex with label $(v) \in \operatorname{Dom}\left(A_{i}\right)$ (resp. label $\left.\left(v^{\prime}\right) \in \operatorname{Dom}\left(A_{j}\right)\right)$ and $\mathcal{P}$ be a set of n-ary closed patterns. An undirected so-called CO-OCCURING relationship, denoted by $e=\left(v, v^{\prime}\right)$, exists between $v$ and $v^{\prime}$ if a pattern $P=\left(A_{1}^{P}, \ldots, A_{n}^{P}, C^{P}\right.$, Supp $\left.^{P}\right)$ can be found in $\mathcal{P}$ such that $A_{i} \neq A_{j}$. Some properties are attached with such relationships: Class represents one class value associated with $P$, i.e., Class $\in C^{P}$; tPattern is a list of pattern identifiers associated with the class value Class where $v$ and $v^{\prime}$ co-occur; and $t$ Supp is a list that contains the support of patterns that are stored in tPattern. The set of CO-OCCURING relationships is denoted by $E_{C}$.

Definition 7 (Graph structure). Let $\mathcal{A}$ be a set of attributes and $\mathcal{P}$ be a set of n-ary closed sets. The graph indexing both attribute values and patterns is defined as $G=(V, E)$ with $E=E_{I} \cup E_{C}$.

Example 5. Figure 3 provides a graphical representation of an example graph structure considering two dimensions and a given set of n-ary closed sets.

\subsection{The Context Matching Operation}

The most frequent query that is run to perform classification is to identify patterns that perfectly match a given context $a=\left(a_{1}, \ldots, a_{n}\right)$. Algorithm 3 details 

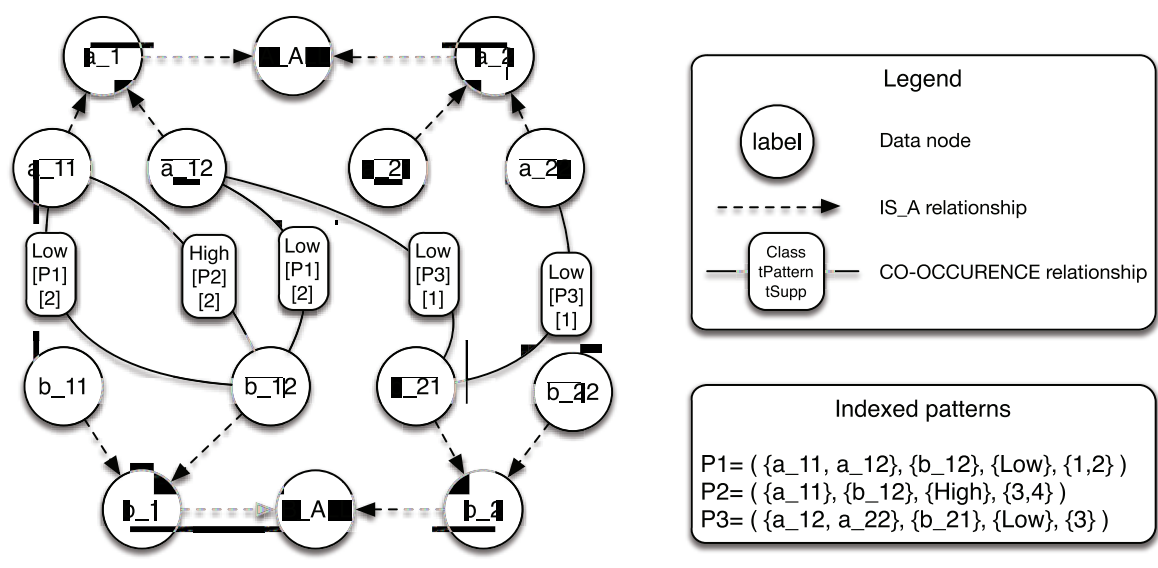

Fig. 3. Graph structure indexing two hierarchical latent attributes and three patterns

the procedure. For ease of reading, it is assumed that we are looking for patterns with one specific class i.e., Class $=c$. The algorithm can, however, be straightforwardly extended to an arbitrary number of classes by repeating this algorithm as many times as the number of class values. Intuitively, the returned set of patterns is the result of the intersection between the edges connecting every pairs of context attribute values. If there exists $a_{i}$ and $a_{j}$ in $a$ such that $v=\left(\operatorname{node}\left(a_{i}\right)\right.$, node $\left.\left(a_{j}\right)\right)$ is not in $V_{C}$, it implies that no pattern match the context $a$ and the algorithm can stop by returning the empty set.

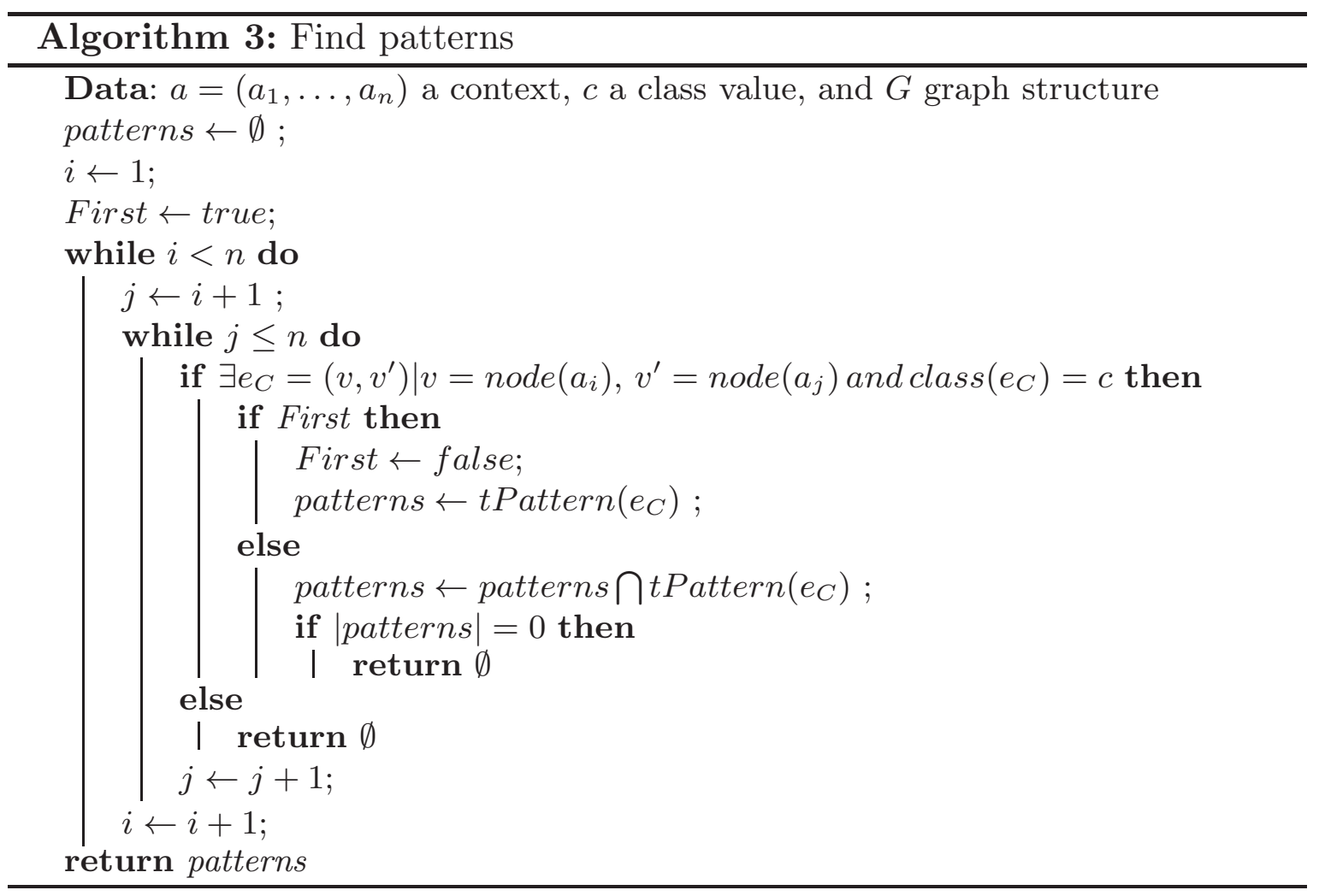




\subsection{Discussion}

The initial requirements described in Section 5.1 are met for two mains reasons. First, from a technology point of view, graph databases are mature enough to be used as an highly performing replacement solution to relational databases. This choice guarantees persistent storage and an efficient query engine [6]. Second, our model leads to a bounded size and a relatively small number of nodes, i.e., the sum of the latent attribute definition domain size. The number of edges is also relatively small, i.e., a few thousands in our real dataset, compared to the the number of mined n-ary closed sets, i.e., up to some millions. These settings enable Algorithm 3 to perform very efficiently as outlined by our experiment results presented in the next section. Finally, we discuss the non-adoption of another more intuitive graph model. In such an alternative model, patterns would be also represented by vertices and a relationship would exist between a data node and a pattern node if the attribute value represented by the data node belongs to the patterns represented by the pattern node. Despite this alternative model is intuitive, it would significantly increase the time needed to classify a single context. Indeed, in this scenario, the number of vertices in the graph database would be huge. Even if modern servers can deal with such amount of data, this point would be very critical regarding the query phase. Indeed, graph databases are not designed to optimally handle so-called super nodes, i.e., nodes having tens of thousands of relationships. This model has been tried out and most of data nodes were indeed super-nodes, i.e., the density of the graph was closed to 1 (complete graph) leading to very slow (up to some minutes) point-wise query response time. Since the time performance of the approach mainly depends on this query response time, the model has been abandoned.

\section{Experimental Evaluation}

This section is dedicated to CnSC evaluation. We first detail the adopted protocol and then present and discuss our result.

\subsection{Protocol}

Dataset. We consider a real dataset from Mobile Broadband Network (MBN). MBN is composed of 600 network nodes (time series producers) which monitor traffic for 5 consecutive weeks at an hourly basis. Traffic load level which represents mobile user activity, allows discretizing nodes into low and high according to traffic level at node. Technical MBN design requires dense node network for high traffic loads, while during low loads some of the MBN nodes potentially could be turned off for a temporal period. MBN traffic classification allows estimating network load at individual network elements and performing network optimization. Three latent attributes and their hierarchical structures are considered: (1) the cell identifier that can be geographically aggregated into sites; (2) the day of the week that is aggregated in either week days or week-end days; 
and (3) the hour that is aggregated in periods, i.e., the night, the morning, the afternoon and the evening.

Evaluation Metrics. From an effectiveness point of view, $\mathrm{CnSC}$ will be evaluated using the standard classification measures, the F-measure. From an efficiency point of view, we will study running time and particularly the model building time, i.e., pattern mining and pattern insertion in the graph database, and the average evaluation time per context to classify.

Competitors. CnSC is compared with a previous work, STEP [13], and the Weka implementation of the Naive Bayes classifier [7].

Evaluated Parameters. We evaluate minSim and minSetSize parameter impact on CnSC. minSim is evaluated in the range from 0.1 to 1 with step of 0.1 (0.8 serves as the default value). minSetSize combinations, i.e., $v_{\text {Strong }}=$

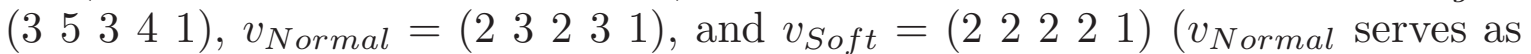
the default value) investigate how the number of mined patters affects results.

Implementation and Validation Process. A 10-fold cross validation strategy has been used. Results presented in this section are thus averaged. The approach has been implemented in Java 1.7. DATA-PEELER software is implemented in $\mathrm{C}++{ }^{4}$. The graph database used in this study is Neo4J (community release, version 2.0 .0$)^{5}$ as it has been showed in [6] that it offers very good performances.

\subsection{Results}

minSetSize Impact. We first evaluate the impact of this parameter on the model construction time (Fig. 4 (left)). It can be decomposed in two main steps: pattern mining and pattern insertion in the database. The less stringent this constraint, the more important the total time. Most of the time is spent to mine patterns. DATA-PEELER extracts around 40M patterns for $v_{\text {Soft }}$, around 10M for $v_{\text {Normal }}$, and only $1 \mathrm{~K}$ for $v_{\text {Strong }}$. Due to a high number of extracted patterns with $v_{S o f t}$, the proposed solution failed maintaining the graph structure. For this reason, setting $v_{\text {Soft }}$ is not evaluated in the remaining experiments. The number of patterns indexed in the graph structure has a big impact on the classification time as stated in Fig. 4 (center). The less patterns, the lower the time to retrieve similar patterns. Moreover, this result shows the great performances of both our algorithms to find similar patterns within the graph and the Neo4j graph databases. Indeed, in the worst case, only $6 \mathrm{~ms}$ are needed to retrieve patterns with similarity greater than 0.8 within the $10 \mathrm{M}$ patterns. From an effectiveness point of view, Fig. 4 (right) shows that the more patterns, the higher the Fmeasure. While F-measure is quite low (0.65) with $v_{\text {Strong }}$, it is significantly better than the two competitors when minSetSize $=v_{\text {Normal }}(0.85$ against 0.81 for STEP and 0.78 for the Naive Bayes classifier).

${ }^{4}$ It can be downloaded at http://homepages.dcc.ufmg.br/ lcerf/fr/prototypes.html.

${ }^{5}$ http://www. neo4j.org 

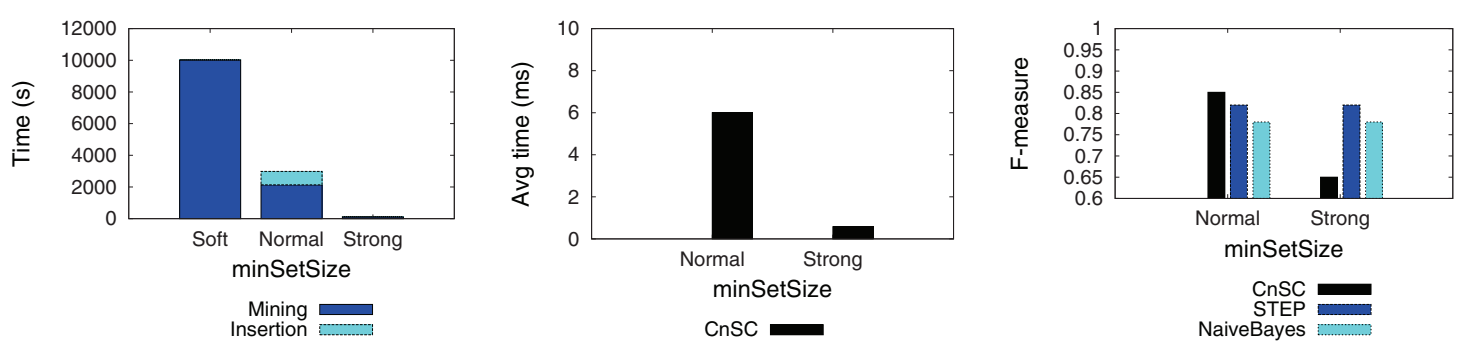

Fig. 4. minSetSize impact: decomposition of the running time during the model construction (left); average time to classify a single context (center); F-measure (right)
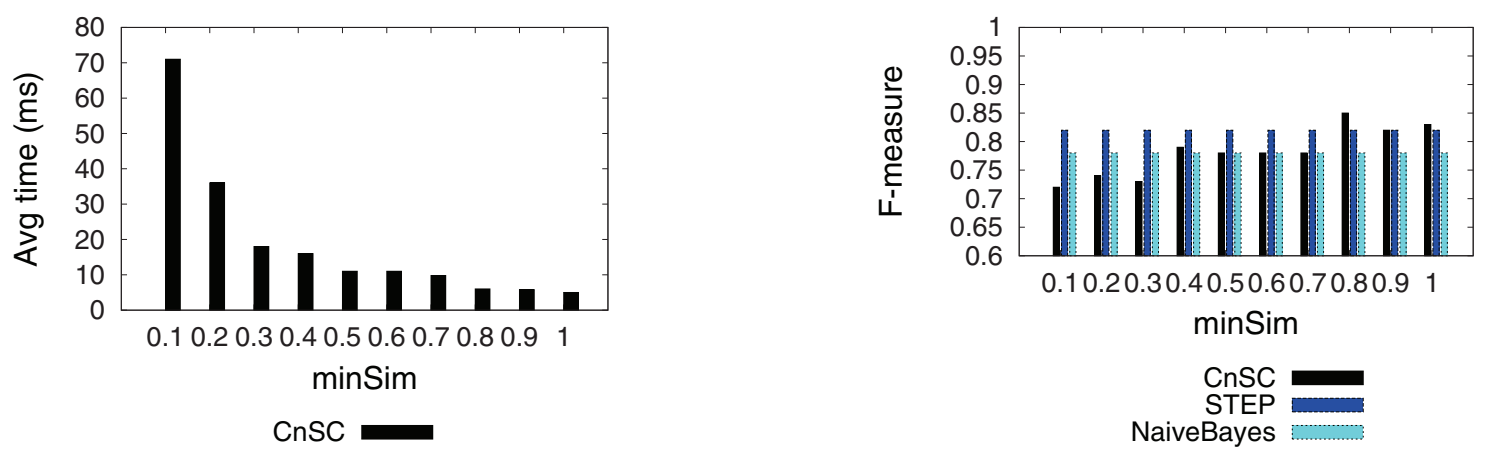

Fig. 5. minSim impact: average time to classify a single context(right); F-measure(left)

minSim Impact. The average time required to classify a context is shown in Fig. 5 (left). Good performances of the average classification time are confirmed. Moreover, as it can be expected, when similarity goes lower, more patterns need to be considered, leading to higher, though acceptable, average classification time. From an effectiveness point of view, two main conclusions can be drawn from Fig. 5 (right). First, when high minSim values are used, too few patterns are considered. This proves the usefulness of taking hierarchies into account to enlarge the context to also include similar ones. Conversely, too low minSim values leads to consider non relevant patterns in the classification process and reduces CnSC accuracy. From Fig. 5 (right) it can be seen that optimal value is minSim $=0.8$. Second, overall performances of CnSC are comparable for most settings and are significantly better when using the optimal value of minSim.

\section{Conclusion}

This paper addressed the problems of classifying time series data and presented CnSC, a novel pattern-based approach. Indeed, closed n-ary sets are extracted from latent attributes to serve as maximal context to describe the class value. Hierarchical aspect of latent attributes is considered to incorporate similar contexts within the classification process. Use of the graph structure and NoSQL graph database allows a very efficient pattern management and classification time. With a good parameter setup, i.e., minSim $=0.8$ and $\operatorname{minSetSize}=v_{\text {Normal }}$, $\mathrm{CnSC}$ offers a significantly better accuracy than two state-of-the-art approaches. 
Several directions can be taken to extend this work. Among them, we can mention automatic estimation of the best parameter values and concept drift detection in the time series distribution to trigger the classification model reconstruction.

\section{References}

1. Bringmann, B., Nijssen, S., Zimmermann, A.: Pattern-based classification: A unifying perspective. CoRR, abs/1111.6191 (2011)

2. Bringmann, B., Zimmermann, A.: One in a million: picking the right patterns. Knowl. Inf. Syst. 18(1), 61-81 (2009)

3. Cerf, L., Besson, J., Robardet, C., Boulicaut, J.-F.: Closed patterns meet n-ary relations. ACM Trans. Knowl. Discov. Data 3(1), 1-3 (2009)

4. Dong, G., Li, J.: Efficient mining of emerging patterns: Discovering trends and differences. In: Proceedings of the Fifth ACM SIGKDD International Conference on Knowledge Discovery and Data Mining, pp. 43-52 (1999)

5. González, A.B., Ramírez Uresti, J.A.: Strategy patterns prediction model (SPPM). In: Batyrshin, I., Sidorov, G. (eds.) MICAI 2011, Part I. LNCS, vol. 7094, pp. 101-112. Springer, Heidelberg (2011)

6. Holzschuher, F., Peinl, R.: Performance of graph query languages: Comparison of cypher, gremlin and native access in neo4j. In: Proceedings of the Joint EDBT/ICDT 2013 Workshops, pp. 195-204 (2013)

7. John, G.H., Langley, P.: Estimating continuous distributions in bayesian classifiers. In: Eleventh Conference on Uncertainty in Artificial Intelligence, pp. 338-345 (1995)

8. Li, W., Han, J., Pei, J.: Cmar: accurate and efficient classification based on multiple class-association rules. In: Proceedings of the 2001 IEEE International Conference on Data Mining, pp. 369-376 (2001)

9. Liu, B., Hsu, W., Ma, Y.: Integrating classification and association rule mining. In: Proceedings of the Fourth International Conference on Knowledge Discovery and Data Mining, pp. 80-86 (1998)

10. Monreale, A., Pinelli, F., Trasarti, R., Giannotti, F.: Wherenext: A location predictor on trajectory pattern mining. In: Proceedings of the 15th ACM SIGKDD International Conference on Knowledge Discovery and Data Mining, pp. 637-646 (2009)

11. Quinlan, J.R.: C4. 5: programs for machine learning, vol. 1. Morgan Kaufmann (1993)

12. Rao, D., Yarowsky, D., Shreevats, A., Gupta, M.: Classifying latent user attributes in twitter. In: Proceedings of the 2nd International Workshop on Search and Mining User-generated Contents, pp. 37-44 (2010)

13. Samulevicius, S., Pitarch, Y., Pedersen, T.B., Sørensen, T.B.: Spatio-temporal ensemble prediction on mobile broadband network data. In: 2013 IEEE 77th Vehicular Technology Conference, pp. 1-5 (2013)

14. Wang, J., Karypis, G.: Harmony: Efficiently mining the best rules for classification. In: Proceedings of the Fifth SIAM International Conference on Data Mining, pp. 205-216

15. Yavas, G., Katsaros, D., Ulusoy, Ö., Manolopoulos, Y.: A data mining approach for location prediction in mobile environments. Data Knowl. Eng. 54(2), 121-146 (2005) 